\title{
The Property of the Set of Equilibria of the Equilibrium Problem with Lower and Upper Bounds on Hadamard Manifolds
}

\author{
Qing-Bang Zhang' and Gusheng Tang ${ }^{2}$ \\ ${ }^{1}$ College of Economic Mathematics, Southwestern University of Finance and Economics, Chengdu, Sichuan 611130, China \\ ${ }^{2}$ School of Mathematics and Computing Science, Hunan University of Science and Technology, Xiangtan, Hunan 411201, China \\ Correspondence should be addressed to Qing-Bang Zhang; zhangqingbang@126.com
}

Received 19 February 2014; Accepted 1 April 2014; Published 30 April 2014

Academic Editor: Xie-ping Ding

Copyright (C) 2014 Q.-B. Zhang and G. Tang. This is an open access article distributed under the Creative Commons Attribution License, which permits unrestricted use, distribution, and reproduction in any medium, provided the original work is properly cited.

The existence of equilibrium points, and the essential stability of the set of equilibrium points of the equilibrium problem with lower and upper bounds are studied on Hadamard manifolds.

\section{Introduction}

Let $K$ be a given nonempty set, $f: K \times K \rightarrow R$ a given function, and $a$ and $b$ two real numbers satisfying $a \leq b$. The equilibrium problem with lower and upper bounds is that of finding $\bar{x} \in K$ such that

$$
a \leq f(\bar{x}, y) \leq b, \quad \forall y \in K .
$$

If $a=0, b=1$, and $f(x, y)=e^{-F(x, y)}$, then problem (1) is said to be the scalar equilibrium problem: find $\bar{x} \in K$ such that

$$
F(\bar{x}, y) \geq 0, \quad \forall y \in K,
$$

where $F: K \times K \rightarrow R$ is a given function satisfying $F(x, x) \geq$ 0 for all $x \in K$. It is well known that problem (2) is a unified model of several problems, such as variational inequality problems, optimization problems, saddle point problems, complementarity problems, and fixed point problems (e.g., see $[1-3])$.

In 1999, Isac et al. [4] raised the open problem: if $K$ is a nonempty closed subset in a locally convex semireflexive topological vector space, under what conditions does problem (1) have a solution? Since then, some authors begin to study the problem. In 2000, Li [5] gave the answer by using the concept of extremal subsets. In [6], Chadli et al. derived some results by using a fixed point theorem due to Ansari and Yao [7] and Fan lemma [8]. In [9], Zhang also answered the problem by using the concept of $(a, b)$-convexity, a fixed point theorem and Fan lemma. The results mentioned above and others in [10-12] are shown in the topological vector space. Therefore, there is a problem: when does problem (1) have a solution in the nonlinear framework of manifolds? On the other hand, as far as we know, there is not a paper in which the essential stability of the set of equilibrium points of the equilibrium problem with lower and upper bounds is given either in topological vector space or on manifolds.

The purpose of this paper is to develop the equilibrium problem with lower and upper bounds in the nonlinear framework of Hadamard manifolds, to study the existence of equilibrium points, and the essential stability of the set of equilibrium points of the equilibrium problem with lower and upper bounds on Hadamard manifolds. Our results extend the corresponding theorems due to Isac et al. [4], Colao et al. [13], and Zhang [9].

\section{Preliminaries}

In this section we recall some notations, definitions, and basic properties used throughout the paper, which can be found in [14] or [15].

Definition 1. A Hadamard manifold $M$ is a complete simply connected Riemannian manifold of nonpositive sectional curvature. 
Throughout this paper, let $M$ be an $m$-dimensional Hadamard manifold, let $x$ be any given point in $M$, and let $T_{x} M$ denote the tangent space at $x$ to $M$. We denote by $\langle\cdot, \cdot\rangle$ the scalar product on $T_{x} M$ with the associated norm $\|\cdot\|$. Let $d: M \times M \rightarrow R$ be the distance function; then by the HopfRinow theorem (see $[15]),(M, d)$ is a complete metric space.

Definition 2. The exponential mapping $\exp _{x}: T_{x} M \rightarrow M$ at $x$ is defined by $\exp _{x} v=\gamma_{v}(1, x)$ for each $v \in T_{x} M$, where $\gamma_{v}(\cdot, x)=\gamma(\cdot)$ is the geodesic starting at $x$ with velocity $v$ (i.e., $\gamma(0)=x$ and $\left.\gamma^{\prime}(0)=v\right)$.

Easily, we know that (i) $\exp _{x} t v=\gamma_{v}(t, x)$ for each real number $t$; (ii) the exponential mapping and its inverse are continuous on Hadamard manifolds; (iii) for any $o, p, q \in M$, the minimal geodesic joining $p$ to $q$ is $\exp _{p} t \exp _{p}^{-1} q$ ( $t \in$ $[0,1])$, and $\exp _{o}\left(t_{1} \exp _{o}^{-1} p+t_{2} \exp _{o}^{-1} q\right)$ is also the minimal geodesic for any $t_{1}, t_{2} \in[0,1]$ with $t_{1}+t_{2}=1$.

Definition 3. A subset $S \subset M$ is said to be geodesic convex if for any two points $x$ and $y$ in $S$ the geodesic joining $x$ to $y$ is contained in $S$; that is, if $\gamma:[a, b] \rightarrow M$ is a geodesic such that $x=\gamma(a)$ and $y=\gamma(b)$, then $\gamma((1-t) a+t b) \in S$ for all $t \in[0,1]$.

Definition 4. Let $o$ be any given point in $M$. The geodesic convex hull for a set $S \subset M$, denoted by $G C o S$, is defined as follows:

$$
\begin{aligned}
G C o S=\left\{\exp _{o}\left(\sum_{i=1}^{n} \lambda_{i} \exp _{o}^{-1} x_{i}\right) \mid\right. & \forall x_{1}, x_{2}, \ldots, x_{n} \in S, \\
& \lambda_{1}, \ldots, \lambda_{n} \in[0,1], \\
& \left.\sum_{i=1}^{n} \lambda_{i}=1\right\} .
\end{aligned}
$$

Remark 5. If $S \subset M$ is a geodesic convex subset, then $G C o\left\{x_{1}, x_{2}, \ldots, x_{n}\right\} \subset S$ for any $x_{1}, x_{2}, \ldots, x_{n} \in S$.

Definition 6 (see [16]). Let $G: S \rightarrow 2^{M}$. One says that $G$ is a KKM mapping on Hadamard manifolds if, for any $x_{1}, x_{2}, \ldots, x_{n} \in S$, one has

$$
G C o\left\{x_{1}, x_{2}, \ldots, x_{n}\right\} \subset \bigcup_{i=1}^{n} G\left(x_{i}\right) .
$$

Lemma 7 (see $[13,16]$ ). Let $K$ be a nonempty closed geodesic convex subset of $M$ and $G: K \rightarrow 2^{M}$ a closed-valued KKM mapping on a Hadamard manifold. If there exists at least one $x_{0} \in K$ such that $G\left(x_{0}\right)$ is compact in $2^{M}$, then

$$
\bigcap_{x \in K} G(x) \neq \emptyset \text {. }
$$

\section{Existence of Equilibrium Point}

In this section, we show the existence of equilibrium point of the equilibrium problem with lower and upper bounds by using KKM theorem on Hadamard manifolds.
Theorem 8. Let $S$ be a nonempty bounded closed and geodesic convex subset of Hadamard manifolds $M$. If the function $f$ : $S \times S \rightarrow R$ satisfies the following conditions:

(i) for each $y \in S$, the set $\{x \in S \mid a \leq f(x, y) \leq b\}$ is closed in $M$,

(ii) for any finite set $\left\{y_{1}, \ldots, y_{n}\right\} \subset S, G \operatorname{Co}\left\{y_{1}, \ldots, y_{n}\right\} \subset$ $\bigcup_{i=1}^{n}\left\{x \in S \mid a \leq f\left(x, y_{i}\right) \leq b\right\}$,

(iii) there exists $y_{0} \in S$, such that $\left\{x \in S \mid a \leq f\left(x, y_{0}\right) \leq b\right\}$ is a compact subset of $M$,

then the equilibrium point of the problem (1) exists. That is, there exists $\bar{x} \in S$, such that

$$
a \leq f(\bar{x}, y) \leq b \quad \forall y \in S .
$$

Proof. Let the set-valued mapping $G: S \rightarrow 2^{S}$ be defined by $G(y)=\{x \in S \mid a \leq f(x, y) \leq b\}$. Then $G$ is a KKM mapping. In fact, it follows from Condition (ii) that, for any finite set $\left\{y_{1}, \ldots, y_{n}\right\} \subset S$ and any $y_{0} \in G \operatorname{Co}\left\{y_{1}, \ldots, y_{n}\right\}$, there exists some $i \in\{1,2, \ldots, n\}$ such that $a \leq f\left(y_{0}, y_{i}\right) \leq b$; that is, $y_{0} \in G\left(y_{i}\right)$ for some $i$. Hence we have $y_{0} \in \bigcup_{i=1}^{n} G\left(y_{i}\right)$ and $\operatorname{Co}\left\{y_{1}, \ldots, y_{n}\right\} \subset \bigcup_{i=1}^{n} G\left(y_{i}\right)$.

By Condition (i), for each $y \in S, G(y)$ is closed in $M$. By Condition (iii) and the completeness of $M$, there exists $y_{0} \in S$ such that $G\left(y_{0}\right)=\left\{x \in S \mid a \leq f\left(x, y_{0}\right) \leq b\right\}$ is compact. By Lemma 7, we have $\bigcap_{y \in S} G(y) \neq \emptyset$; that is, for any $y \in S$, there exist $\bar{x} \in G(y)$. Therefore there exists $\bar{x} \in S$ such that $a \leq f(\bar{x}, y) \leq b$ for all $y \in S$. The proof is completed.

Example 9. If for any $x \in S$, the mapping $g: S \times S \rightarrow R$ satisfies that the set $\{y \in S \mid g(x, y)<0\}$ is geodesic convex and $g(x, x)=0$, then $f(x, y)=e^{-g(x, y)}$ satisfies Condition (ii).

In fact, if not, then for any finite set $\left\{y_{1}, \ldots, y_{n}\right\} \subset S$, there exists $\widehat{x} \in G \operatorname{Co}\left\{y_{1}, \ldots, y_{n}\right\}$ such that $\widehat{x} \notin \bigcup_{i=1}^{n}\{x \in S$ । $\left.g\left(x, y_{i}\right) \geq 0\right\}$; that is, $g\left(\hat{x}, y_{i}\right)<0$ (for all $i \in\{1,2, \ldots, n\}$ ). This implies that for any $i \in\{1,2, \ldots, n\}, y_{i} \in\{y \in S \mid$ $g(\widehat{x}, y)<0\}$. By the geodesic convexity of $\{y \in S \mid g(x, y)<$ $0\}$, we have $\widehat{x} \in G C o\left\{y_{1}, \ldots, y_{n}\right\} \subset\{y \in S \mid g(\widehat{x}, y)<0\}$, which contradicts to $g(x, x)=0$. Therefore, for any subset $\left\{y_{1}, \ldots, y_{n}\right\} \subset S, G \operatorname{Co}\left\{y_{1}, \ldots, y_{n}\right\} \subset \bigcup_{i=1}^{n}\left\{x \in S \mid g\left(x, y_{i}\right) \geq\right.$ $0\} \subset \bigcup_{i=1}^{n}\left\{x \in S \mid 0 \leq f\left(x, y_{i}\right) \leq 1\right\}$.

Theorem 10. Let $S$ be a nonempty bounded closed and geodesic convex subset of Hadamard manifolds $M$. If the function $f$ : $S \times S \rightarrow R$ satisfies the following conditions:

(i) for each $y \in S$, the set $\{x \in S \mid a \leq f(x, y) \leq b\}$ is closed in $M$,

(ii) for any finite set $\left\{y_{1}, \ldots, y_{n}\right\} \subset S, G \operatorname{Co}\left\{y_{1}, \ldots, y_{n}\right\} \subset$ $\bigcup_{i=1}^{n}\left\{x \in S \mid a \leq f\left(x, y_{i}\right) \leq b\right\}$,

(iii) there exists a compact subset $L \subset M$ and a point $y_{0} \in$ $L \cap S$, such that $f\left(x, y_{0}\right)<a$ or $f\left(x, y_{0}\right)>b$ for all $x \in S \backslash L$,

then the equilibrium point of problem (1) exists.

Proof. Let the set-valued mapping $G: S \rightarrow 2^{S}$ be defined by $G(y)=\{x \in S \mid a \leq f(x, y) \leq b\}$. Then by Condition 
(iii) there exists a point $y_{0} \in S$ such that $G\left(y_{0}\right) \subset L$. So it follows for Condition (i) and the completeness of $M$ that $G\left(y_{0}\right)$ is compact. By Theorem 8 , we have that there exists $\bar{x} \in S$ such that $a \leq f(\bar{x}, y) \leq b$ for all $y \in S$. This completes the proof.

Theorem 11. Let $S$ be a nonempty compact and geodesic convex subset of Hadamard manifolds $M$. If the function $f: S \times$ $S \rightarrow R$ satisfies the following conditions:

(i) for each $y \in S, f$ is continuous with respect to $x$ in $M$,

(ii) for any finite set $\left\{y_{1}, \ldots, y_{n}\right\} \subset S, G C o\left\{y_{1}, \ldots, y_{n}\right\} \subset$ $\bigcup_{i=1}^{n}\left\{x \in S \mid a \leq f\left(x, y_{i}\right) \leq b\right\}$,

then there exists $\bar{x} \in S$, such that $a \leq f(\bar{x}, y) \leq b$ for all $y \in S$.

Proof. From the continuity of $f$, it follows that Condition (i) of Theorem 8 holds. By Theorem 8 , we have that there exists $\bar{x} \in S$ such that $a \leq f(\bar{x}, y) \leq b$ for all $y \in S$. This completes the proof.

Remark 12. Theorem 8 extends Theorem 3.1 due to Zhang [9] from the topological vector space to Hadamard manifolds.

Next, we show some applications of our results as the following.

Corollary 13. Let $S$ be a nonempty compact and geodesic convex subset of Hadamard manifolds $M, a=\inf _{x \in S} f(x, x)$, and $b=\sup _{x \in S} f(x, x)$. If the function $f: S \times S \rightarrow R$ satisfies the following conditions:

(i) for each $y \in S, f$ is continuous with respect to $x$ in $M$,

(ii) for any finite set $\left\{y_{1}, \ldots, y_{n}\right\} \subset S, G C o\left\{y_{1}, \ldots, y_{n}\right\} \subset$ $\bigcup_{i=1}^{n}\left\{x \in S \mid a \leq f\left(x, y_{i}\right) \leq b\right\}$,

then there exists $\bar{x} \in S$, such that

$$
\inf _{x \in S} f(x, x) \leq f(\bar{x}, y) \leq \sup _{x \in S} f(x, x), \quad \forall y \in S .
$$

Corollary 14. Let $S$ be a nonempty bounded closed and geodesic convex subset of Hadamard manifolds $M$. If the mapping $F: S \times S \rightarrow R$ satisfies the following conditions:

(i) for any $x \in S, F(x, x)=0$,

(ii) for each $y \in S$, the set $\{x \in S \mid F(x, y) \geq 0\}$ is closed in $M$,

(iii) for any $x \in S$, the set $\{y \in S \mid F(x, y)<0\}$ is geodesic convex,

(iv) there exists a compact subset $L \subset M$ and a point $y_{0} \in$ $L \cap S$, such that $F\left(x, y_{0}\right)<0$ for all $x \in S \backslash L$,

then the equilibrium point of the problem (2) exists.

Proof. Define a mapping $f: S \times S \rightarrow R$ by $f(x, y)=e^{-F(x, y)}$, and then

$$
\{x \in S \mid F(x, y) \geq 0\}=\{x \in S \mid 0<f(x, y) \leq 1\} .
$$

By Condition (ii), we have that the $\{x \in S \mid 0 \leq f(x, y) \leq 1\}$ is closed. It follows from conditions (i), (iii) and Example 9 that $f$ satisfies that for any finite set $\left\{y_{1}, \ldots, y_{n}\right\} \subset S$, $G \operatorname{Co}\left\{y_{1}, \ldots, y_{n}\right\} \subset \bigcup_{i=1}^{n}\left\{x \in S \mid 0 \leq f\left(x, y_{i}\right) \leq 1\right\}$. Additionally, by Condition (iv) there exists a point $y_{0} \in S$ for which $\left\{x \in S \mid F\left(x, y_{0}\right) \geq 0\right\} \subset L$. So it follows from Condition (ii) and the completeness of $M$ that $\{x \in S \mid 0 \leq f(x, y) \leq 1\}$ is compact. By Theorem 8 , we have that, for all $y \in S$, there exists $\bar{x} \in S$ such that $0 \leq f(\bar{x}, y) \leq 1$; that is, there exists $\bar{x} \in S$ such that $F(x, y) \geq 0$. This completes the proof.

Remark 15. When the mapping $x \rightarrow F(x, y)$ is upper continuous for all $y \in S$, Condition (i) holds in Corollary 14. If $S$ is a compact subset of $M$, then Condition (iv) can be omitted. Therefore, Theorem 3.2 shown in [13] is improved.

\section{Essential Stability}

In this section, we consider the essential stability of the set of equilibrium points of the equilibrium problem with lower and upper bounds on Hadamard manifolds. We can see the systemic study about the essential stability in the topological vector space in [17].

Let $S$ be a nonempty compact and geodesic convex subset of Hadamard manifold $M$, and $\Psi$ denotes the set of the function $f: S \times S \rightarrow R$, which is continuous with respect to $x$ and satisfies $G \operatorname{Co}\left\{y_{1}, \ldots, y_{n}\right\} \subset \bigcup_{i=1}^{n}\left\{x \in S \mid a \leq f\left(x, y_{i}\right) \leq\right.$ $b\}$ for any finite set $\left\{y_{1}, \ldots, y_{n}\right\} \subset S$.

For any $f \in \Psi$, it follows from Theorem 8 that there exists $\bar{x} \in S$ such that for all $y \in S, a \leq f(\bar{x}, y) \leq b$, where $\bar{x} \in$ $S$ is said to be equilibrium points of the equilibrium problem with lower and upper bounds. Let $E(f)$ denote the set of equilibrium points $\bar{x}$; then

$$
E(f)=\left\{\bar{x} \in S \mid \bar{x} \in \bigcap_{y \in S}\{x \mid a \leq f(x, y) \leq b\}\right\} .
$$

So a mapping $E: \Psi \rightarrow K(S)$ is well defined, where $K(S)$ is the set of all nonempty compact subsets of $S$. For any $f, g \in \Psi$, we can define a distance as follows:

$$
\rho(f, g)=\sup _{(x, y) \in S \times S}|f(x, y)-g(x, y)| .
$$

Clearly, $(\Psi, \rho)$ is a metric space.

Definition 16. For each $f \in \Psi$, let $e(f)$ be a nonempty closed subset of $E(f)$.

(i) $x \in E(f)$ is called an essential point of $E(f)$ if, for any open neighborhood $N(x)$ of $x$ in $S$, there is a $\delta>0$ such that for any $f^{\prime} \in \Psi$ with $\rho\left(f, f^{\prime}\right)<\delta, N(x) \cap$ $E\left(f^{\prime}\right) \neq \emptyset$. If all $x \in E(f)$ is essential, then $f$ is said to be essential.

(ii) $e(f)$ is called an essential set of $E(f)$ if, for any open set $U, e(f) \subset U$, there is a $\delta>0$ such that for any $f^{\prime} \in \Psi$ with $\rho\left(f, f^{\prime}\right)<\delta, U \cap E\left(f^{\prime}\right) \neq \emptyset$.

(iii) $m(f)$ is called a minimal essential set of $E(f)$ if it is a minimal element of the family of essential sets ordered by set inclusion. 
Lemma 17. The metric space $(\Psi, \rho)$ is complete.

Proof. Let $\left\{f_{n} \in \Psi\right\}$ be any Cauchy sequence; then for any $\varepsilon>0$, there is an $n_{0} \in N$ such that for any $n, m>n_{0}$, $\rho\left(f_{n}, f_{m}\right)<\varepsilon$, or, $\sup _{(x, y) \in S \times S}\left|f_{n}(x, y)-f_{m}(x, y)\right|<\varepsilon$, which implies that for any $(x, y) \in S \times S,\left\{f_{n}(x, y)\right\}$ is a Cauchy sequence in $R$. Thus, there is a mapping $f: S \times S \rightarrow R$ such that $\left|f_{n}(x, y)-f(x, y)\right| \rightarrow 0$ for each $(x, y) \in S \times S$. Hence $\sup _{(x, y) \in S \times S}\left|f_{n}(x, y)-f(x, y)\right| \rightarrow 0$. Then $f_{n} \rightarrow f$ under the metric $\rho$.

Next we will prove $f \in \Psi$. For any $y \in S$, using

$$
\begin{aligned}
& \left|f\left(x_{n}, y\right)-f(x, y)\right| \\
& \quad \leq\left|f_{n}\left(x_{n}, y\right)-f\left(x_{n}, y\right)\right| \\
& \quad+\left|f_{n}(x, y)-f(x, y)\right|+\left|f_{n}\left(x_{n}, y\right)-f_{n}(x, y)\right|
\end{aligned}
$$

we can show $f(x, y)$ is continuous with respect to $x$. For all finite set $\left\{y_{1}, \ldots, y_{n}\right\} \subset S$ and all $y_{0} \in G C o\left\{y_{1}, \ldots, y_{n}\right\}$, it follows from the property of $f_{n}$ that there exists some $i \in$ $\{1,2, \ldots, n\}$ such that $a \leq f_{n}\left(y_{0}, y_{i}\right) \leq b$. Since $f_{n} \rightarrow f$ under the metric $\rho, a \leq f\left(y_{0}, y_{i}\right) \leq b$ holds for some $y_{i}$ and any $y_{0}$. Then

$$
G C o\left\{y_{1}, \ldots, y_{n}\right\} \subset \bigcup_{i=1}^{n}\left\{x \in S a \leq f\left(x, y_{i}\right) \leq b\right\}
$$

that is, $f \in \Psi$.

Lemma 18. The mapping $E: \Psi \rightarrow K(S)$ is a usco mapping; that is, $E$ is upper semicontinuous on $\Psi$ and $E(f)$ is nonempty compact for all $f \in \Psi$.

Proof. Since $S$ is compact, we need only to prove the closedness of the graph of $E$ (Grap $E)$; that is, for all $f_{n} \in \Psi$ with $f_{n} \rightarrow f$ and any $x_{n} \in E\left(f_{n}\right)$ with $x_{n} \rightarrow x, x \in E(f)$ should be proved.

For any $n, x_{n} \in E\left(f_{n}\right)$ implies that $x_{n} \in \bigcap_{y \in S}\{x \in S \mid a \leq$ $\left.f_{n}(x, y) \leq b\right\}$. Hence, for all $y \in S$, by the continuity of $f_{n}$, we have that $\left\{x \in S \mid a \leq f_{n}(x, y) \leq b\right\}$ is closed, and so $x \in$ $\bigcap_{y \in S}\left\{x \in S a \leq f_{n}(x, y) \leq b\right\}$; that is, $a \leq f_{n}(x, y) \leq b$ holds for all $y \in S$. It follows from $f_{n} \rightarrow f$ that $a \leq f(x, y) \leq b$ holds for all $y \in S$. Therefore, $x \in E(f)$. This completes the proof.

Theorem 19. For each $f \in \Psi$, one has that

(i) there exists a dense residual subset $G$ of $\Psi$ such that for each $f \in G, f$ is essential,

(ii) there exists at least one connected minimal essential subset of $E(f)$.

Proof. (i) By Lemmas 17 and 18, we have that the metric space $(\Psi, \rho)$ is complete and the mapping $E: \Psi \rightarrow K(S)$ is usco. Hence, it follows from Fort theorem (see [18]) that there is a dense residual subset $G$ of $\Psi$, such that $E$ is lower semicontinuous in $G$. From the definition of lower semicontinuous mapping and Definition 16 (i), it follows that $f$ is essential for each $f \in G$.

(ii) Let $\Theta$ denote the family of all essential subsets of $E(f)$ ordered by set inclusion; then $\Theta \neq \emptyset$. In fact, the upper semicontinuity of $E$ implies that, for each open set $\Lambda$ with $E(f) \subset \Lambda$, there exists $\delta>0$ such that for any $f^{\prime} \in \Psi$ with $\rho\left(f, f^{\prime}\right)<\delta, E\left(f^{\prime}\right) \subset \Lambda$. Hence $E(f)$ is an essential set of itself.

From the compactness of $S$, it follows that the intersection of every decreasing chain of elements in $\Theta$ is also in $\Theta$ and $\Theta$ has a lower bound. Therefore, by Zorns lemma, $\Theta$ has a minimal element $m(f)$, which is a minimal essential set of $E(f)$.

Suppose that the minimal essential subset $m(f)$ is not connected. Then, there exist two nonempty open subsets $M_{1}$ and $M_{2}$ with $m(f)=M_{1} \cup M_{2}$ and two disjoint open subsets $V_{1}$ and $V_{2}$ in $E(f)$ such that $V_{1} \supset M_{1}$ and $V_{2} \supset M_{2}$. From $m(f) \subset V_{1} \cup V_{2}$, it follows that there is a $\delta^{*}>0$ such that for any $f^{\prime} \in \Psi$ with $\rho\left(f, f^{\prime}\right)<\delta^{*},\left(V_{1} \cup V_{2}\right) \cap E\left(f^{\prime}\right) \neq \emptyset$.

Since $m(f)$ is a minimal essential set of $E(f)$, then neither $M_{1}$ nor $M_{2}$ is essential. Hence, for $\delta^{*}>0$, there exist $f_{1}, f_{2} \in$ $\Psi$ with $\rho\left(f, f_{1}\right)<\left(\delta^{*} / 4\right)$ and $\rho\left(f, f_{2}\right)<\left(\delta^{*} / 4\right)$, such that $V_{1} \cap E\left(f_{1}\right)=\emptyset, V_{2} \cap E\left(f_{2}\right)=\emptyset$. Thus, $\rho\left(f_{1}, f_{2}\right)<\left(\delta^{*} / 2\right)$.

Define a mapping $f_{3}: S \times S \rightarrow R$ as follows:

$$
f_{3}(x, y)=\alpha(y) f_{1}(x, y)+\beta(y) f_{2}(x, y),
$$

where $\alpha(y)=d\left(y, V_{2}\right) /\left(d\left(y, V_{1}\right)+d\left(y, V_{2}\right)\right), \beta(y)=$ $d\left(y, V_{1}\right) /\left(d\left(y, V_{1}\right)+d\left(y, V_{2}\right)\right)$, and $d(\cdot, \cdot)$ is the metric of $M$. It is easy to show that, for any $y \in S, \alpha(y)$ and $\beta(y)$ are continuous, $\alpha(y) \geq 0, \beta(y) \geq 0$, and $\alpha(y)+\beta(y)=1$. By the continuity of $f_{1}$ and $f_{2}$, we have that $f_{3}$ is continuous with respect to $x$.

For any finite set $\left\{y_{1}, \ldots, y_{n}\right\} \subset S$ and all $y_{0} \in$ $G \operatorname{Co}\left\{y_{1}, \ldots, y_{n}\right\}$, it follows from the property of $f_{j}(j=$ $1,2)$ that there exists some $i \in\{1,2, \ldots, n\}$ such that $a \leq$ $f_{j}\left(y_{0}, y_{i}\right) \leq b(j=1,2)$, and then $a \leq f_{3}\left(y_{0}, y_{i}\right) \leq b$. Therefore, $f_{3} \in \Psi$.

Since

$$
\begin{aligned}
\rho\left(f_{3}, f\right) & \leq \rho\left(f_{3}, f_{1}\right)+\rho\left(f_{1}, f\right) \\
& \leq \rho\left(f_{1}, f\right)+\rho\left(f_{1}, f_{2}\right) \leq \delta^{*},
\end{aligned}
$$

we have $\left(V_{1} \cup V_{2}\right) \cap E\left(f_{3}\right) \neq \emptyset$. When $V_{1} \cap E\left(f_{3}\right) \neq \emptyset$, let $\bar{y} \in V_{1} \cap$ $E\left(f_{3}\right)$; then $\alpha(\bar{y})=1, \beta(\bar{y})=0, f_{3}=f_{1}$, and $V_{1} \cap E\left(f_{1}\right) \neq \emptyset$, which contradicts the fact $V_{1} \cap E\left(f_{1}\right)=\emptyset$. Similarly, we can show that $V_{2} \cap E\left(f_{3}\right) \neq \emptyset$ results in a contradiction. Therefore, $m(f)$ is connected. The proof is completed.

In the sequel, by using the above results, we consider the essential stability of the set of equilibrium points of problem (2) on Hadamard manifolds.

Let $\Psi^{\prime}$ denote the set of the function $F: S \times S \rightarrow R$, which is continuous and satisfies that $\{x \in S \mid F(x, y)<0\}$ is geodesic convex and $F(x, x)=0$ for any $x \in S$.

For any $F \in \Psi^{\prime}$, it follows from Remark 15 and Corollary 14 that there exists $\bar{x} \in S$ such that for all $y \in S$, $F(\bar{x}, y) \geq 0$, where $\bar{x} \in S$ is said to be equilibrium points of 
problem (2). Let $E^{\prime}(F)$ denote the set of equilibrium points $\bar{x}$; then

$$
\begin{aligned}
E^{\prime}(F) & =\left\{\bar{x} \in S \mid \bar{x} \in \bigcap_{y \in S}\{x \mid F(x, y) \geq 0\}\right\} \\
& =\left\{\bar{x} \in S \mid \bar{x} \in \bigcap_{y \in S}\left\{x \mid 0 \leq e^{-F(x, y)} \leq 1\right\}\right\} .
\end{aligned}
$$

So a mapping $E^{\prime}: \Psi^{\prime} \rightarrow K(S)$ is well defined.

Let $f(x, y)=e^{-F(x, y)}, a=0$, and $b=1$; by Theorem 19, we have the following results.

Corollary 20. For each $F \in \Psi^{\prime}$, one has that

(i) there exists a dense residual subset $G^{\prime}$ of $\Psi^{\prime}$ such that for each $F \in G^{\prime}, F$ is essential,

(ii) there exists at least one connected minimal essential subset of $E^{\prime}(F)$.

\section{Conflict of Interests}

The authors declare that there is no conflict of interests regarding the publication of this paper.

\section{Acknowledgments}

The authors are grateful to the referees for their suggestions to improve the paper. This work is supported by National Natural Science Foundation of China (no. 11201379), the Fundamental Research Funds for the Central Universities (no. JBK130401), Scientific Research Fund of SiChuan Provincial Education Department (no. 14ZA0362), and the Natural Science Foundation of Hunan Provincial (no. 2014JJ4044).

\section{References}

[1] E. Blum and W. Oettli, "From optimization and variational inequalities to equilibrium problems," The Mathematics Student, vol. 63, no. 1-4, pp. 123-145, 1994.

[2] H. Brezis, L. Nirenberg, and G. Stampacchia, "A remark on Ky Fan's minimax principle," Bollettino della Unione Matematica Italiana, vol. 6, no. 4, pp. 293-300, 1972.

[3] G. Bigi, M. Castellani, M. Pappalardo, and M. Passacantando, "Existence and solution methods for equilibria," European Journal of Operational Research, vol. 227, no. 1, pp. 1-11, 2013.

[4] G. Isac, V. M. Sehgal, and S. P. Singh, "An alternate version of a variational inequality," Indian Journal of Mathematics, vol. 41, no. 1, pp. 25-31, 1999.

[5] J. Li, "A lower and upper bounds version of a variational inequality," Applied Mathematics Letters, vol. 13, no. 5, pp. 47-51, 2000.

[6] O. Chadli, Y. Chiang, and J. C. Yao, "Equilibrium problems with lower and upper bounds," Applied Mathematics Letters, vol. 15, no. 3, pp. 327-331, 2002.

[7] Q. H. Ansari and J.-C. Yao, "A fixed point theorem and its applications to a system of variational inequalities," Bulletin of the Australian Mathematical Society, vol. 59, no. 3, pp. 433-442, 1999.
[8] K. Fan, "A generalization of Tychonoff's fixed point theorem," Mathematische Annalen, vol. 142, no. 3, pp. 305-310, 1961.

[9] C. Zhang, "A class of equilibrium problems with lower and upper bounds," Nonlinear Analysis: Theory, Methods \& Applications, vol. 63, no. 5-7, pp. e2377-e2385, 2005.

[10] S. Al-Homidan and Q. H. Ansari, "Systems of quasi-equilibrium problems with lower and upper bounds," Applied Mathematics Letters, vol. 20, no. 3, pp. 323-328, 2007.

[11] L. Fan, "Weighted quasi-equilibrium problems with lower and upper bounds," Nonlinear Analysis: Theory, Methods \& Applications, vol. 70, no. 6, pp. 2280-2287, 2009.

[12] Z. D. Mitrovi and M. Merkle, "On a generalized vector equilibrium problem with bounds," Applied Mathematics Letters, vol. 23, no. 7, pp. 783-787, 2010.

[13] V. Colao, G. López, G. Marino, and V. Martín-Márquez, "Equilibrium problems in Hadamard manifolds," Journal of Mathematical Analysis and Applications, vol. 388, no. 1, pp. 61-77, 2012.

[14] M. P. DoCarmo, Riemannian Geometry, Birkhäuser, Boston, Mass, USA, 1992.

[15] I. Chavel, Riemannian Geometry: A Modern Introduction, vol. 108, Cambridge University Press, Cambridge, Mass, USA, 1993.

[16] L. W. Zhou and N. J. Huang, "Generalized KKM theorems on Hadamard manifolds with applications," 2009, http://www.paper.edu.cn/index.php/default/releasepaper/content/200906-669.

[17] J. Yu, Game Theory and Nonlinear Analysis, Science Press, Beijing, China, 2008 (Chinese).

[18] M. K. Fort, Jr., "Essential and non essential fixed points," American Journal of Mathematics, vol. 72, no. 2, pp. 315-322, 1950. 


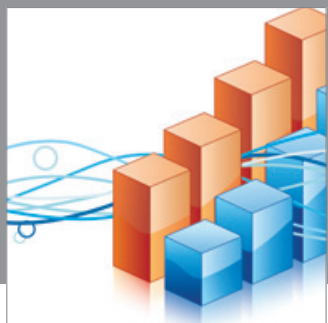

Advances in

Operations Research

mansans

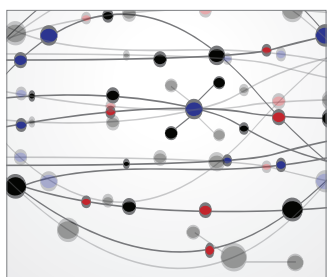

The Scientific World Journal
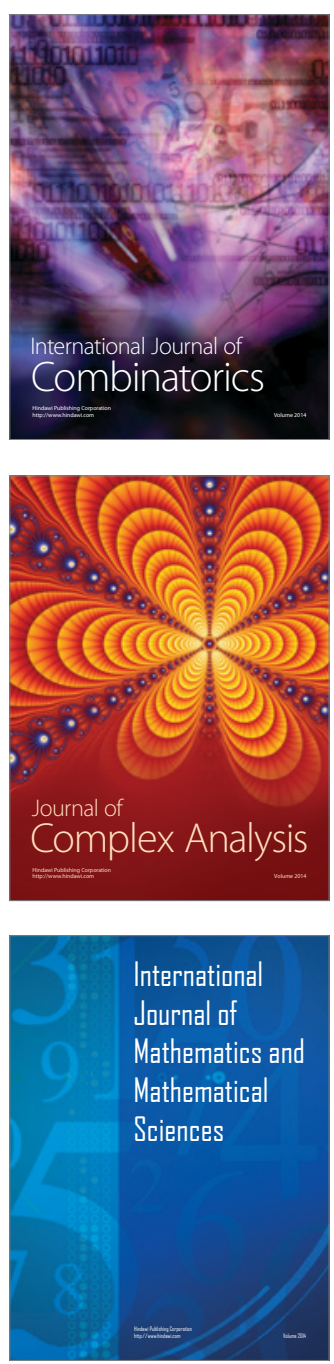
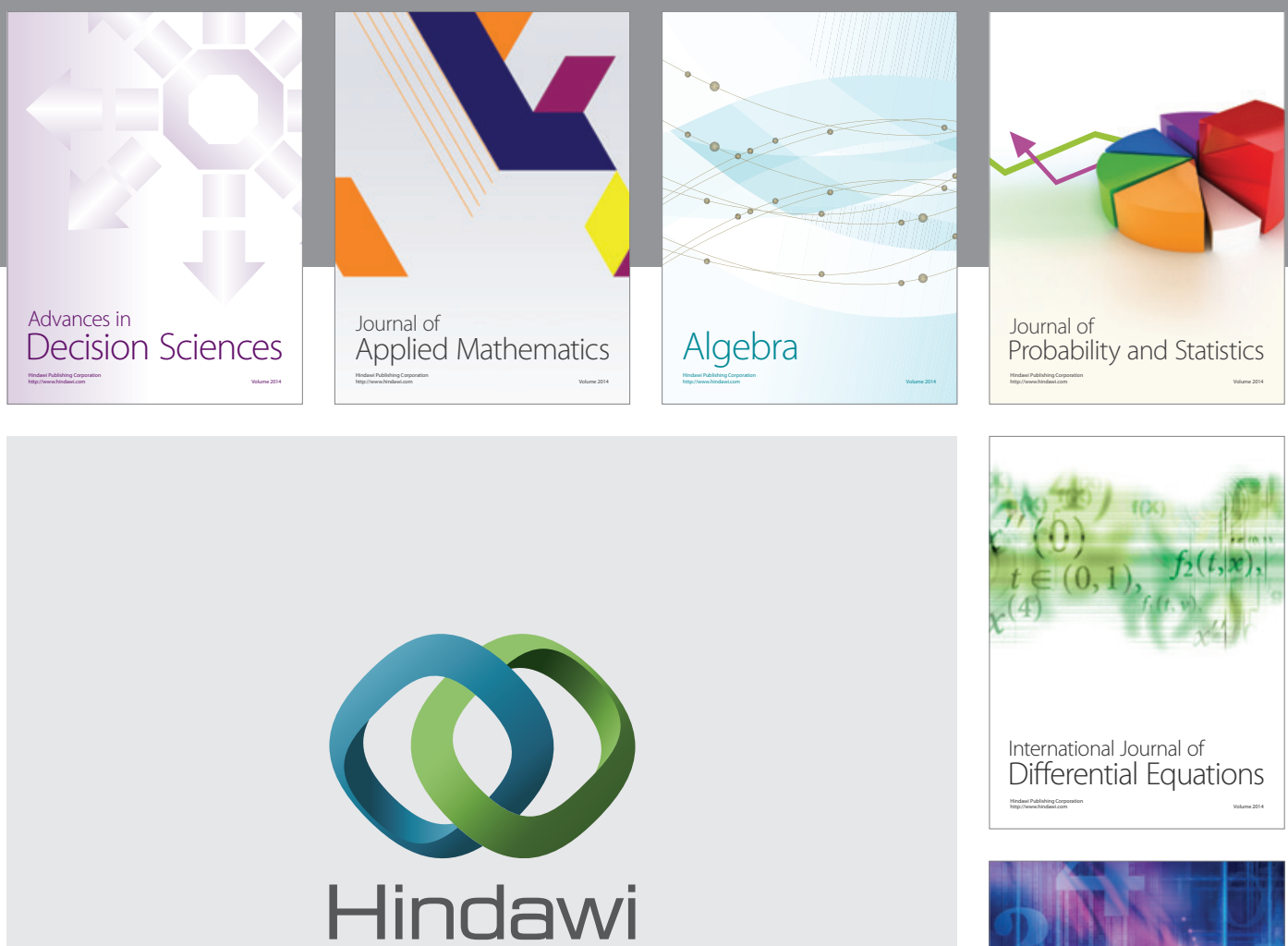

Submit your manuscripts at http://www.hindawi.com
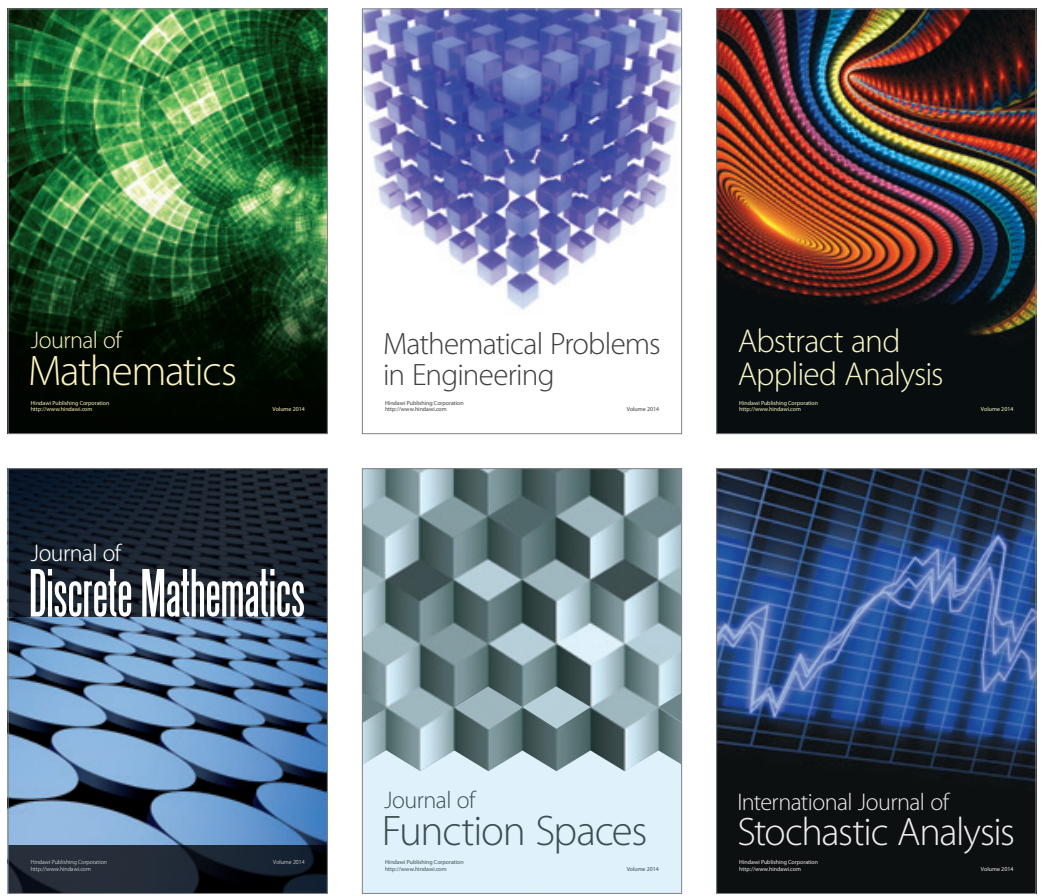

Journal of

Function Spaces

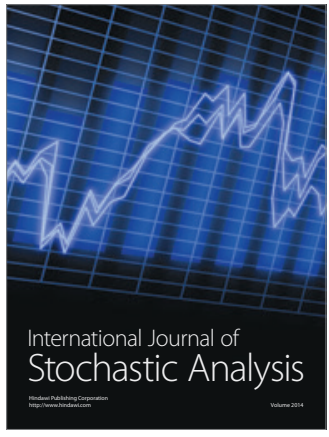

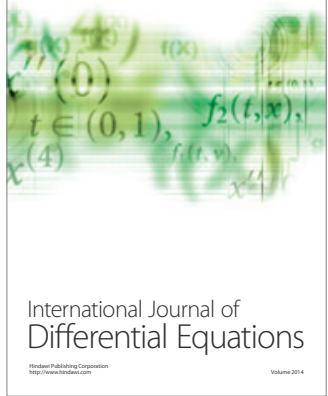
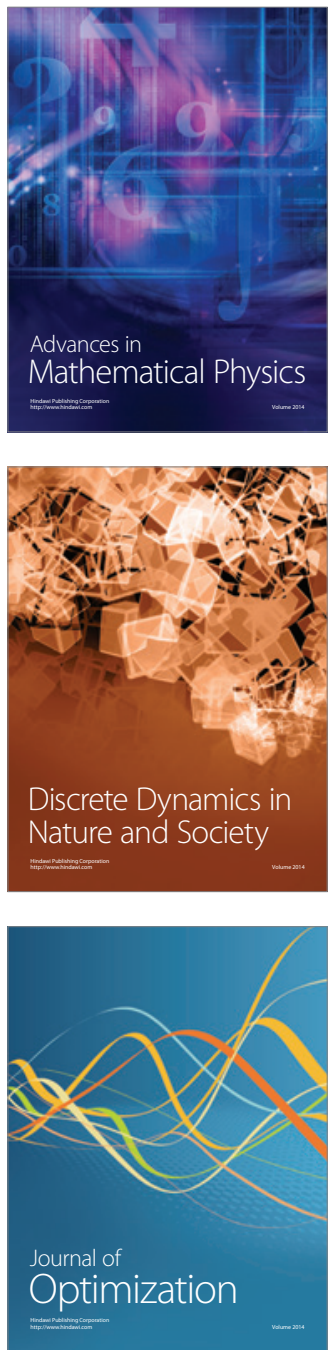